\title{
Comparisons of clinically based outcome measures and laboratory-based outcome measure for balance in patients following total hip and knee arthroplasty
}

This article was published in the following Dove Press journal:

Orthopedic Research and Reviews

10 April 2017

Number of times this article has been viewed

\section{Pankaj Jogi \\ Tom Overend \\ John Kramer}

School of Physical Therapy, University of Western Ontario, London, ON, Canada

\section{Video abstract}

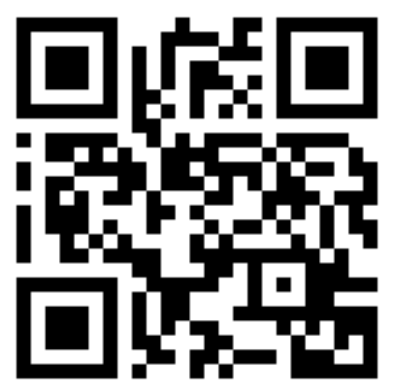

Point your SmartPhone at the code above. If you have a QR code reader the video abstract will appear. Or use: http://youtu.be/q_F7WrlauX4
Correspondence: Pankaj Jogi

School of Physical Therapy, University of Western Ontario, London, ON N6G

IHI, Canada

Tel +l 2029992645

Email pankaj_jogi@hotmail.com
Background: Information available in the literature on clinically based and laboratory-based outcome measures of balance is limited. How much information is provided by clinically based outcome measures compared to laboratory-based measure in patients with total hip (THA) and knee arthroplasty (TKA) is not known.

Aim: The purpose of this study was to determine the relationship between selected clinically based outcome measures and laboratory-based force platform measure in patients following THA and TKA.

Methods: Patients who underwent THA $(n=26)$ and TKA $(n=28)$ were evaluated at about 5-7 weeks following surgery. Participants were assessed using four clinically based outcome measures - 1) the Berg Balance Scale (BBS), 2) the Timed Up and Go test (TUG), 3) the Activities-specific Balance Confidence Scale (ABC), and 4) the Western Ontario McMaster Universities Osteoarthritis Index-function subscale (WOMAC-function) - and one laboratorybased force plate measure ( $95 \%$ ellipse area).

Results: Moderate correlations were observed between the BBS and the $95 \%$ ellipse area of force plate ( $r=0.46-0.51)$ for the two-legged stance, the anterior lean stance, and the posterior lean stance. Fair correlations were observed between TUG and the 95\% ellipse area of force plate $(r=0.31-0.37)$ for all the three test conditions. Low correlations were observed for the $\mathrm{ABC}$ and the WOMAC-function with the 95\% ellipse area of force plate $(r=0.11-0.25)$ for all the three test conditions.

Conclusion: The BBS demonstrated the greatest correlations with the $95 \%$ ellipse area of the force plate measure and should be preferred by physical therapists over the TUG, the ABC, and the WOMAC-function to assess balance in patients with THA and TKA.

Keywords: total knee arthroplasty, total hip arthroplasty, force plate, clinical measures, balance

\section{Introduction}

Balance is critical for mobility and physical function in the elderly. ${ }^{1,2}$ Balance requires integration of information from somatosensory, visual, and vestibular systems and execution of appropriate musculoskeletal responses throughout the body, including the joints of the lower extremities. ${ }^{3}$ Following orthopedic injury to the joints of the lower extremities, the sensory and motor processes are disrupted subsequently leading to balance problems. ${ }^{4}$ Hip and knee osteoarthritis (OA) has been reported to significantly reduce standing balance in the elderly. ${ }^{5}$ Although interventions such as total hip arthroplasty (THA) and total knee arthroplasty (TKA) for hip and knee OA, respectively, 
help to reduce joint pain and stiffness, and improve physical function, they may not necessarily improve patient's balance. THA and TKA are costly surgeries with respect to actual financial costs for surgery and those incurred for patient care and rehabilitation during the recovery process. It is in the interest of the patient, health care professionals, and service providers that the outcome of THA and TKA is successful. Among many causes for unsuccessful surgery is injury to the replaced hip and knee joint as a result of fall. Impairment in balance is a major cause of falls. Patients with severe hip and knee OA, as well as those who had undergone THA and TKA, have been reported to have a higher incidence of falls compared to the general population. ${ }^{6}$

A variety of approaches that include subjective and objective methods have been used to assess balance in the clinical and research areas. Clinically, several outcome measures have been used to assess balance in the elderly and in patients with THA and TKA. The Berg Balance Scale (BBS) ${ }^{7}$ and the Timed Up and Go test $(\mathrm{TUG})^{8}$ which are clinician administered have been commonly used to assess balance in patients with THA and TKA. The Activities-specific Balance Confidence Scale $(\mathrm{ABC})^{9}$ and the Western Ontario McMaster Universities Osteoarthritis Index (WOMAC) ${ }^{10}$ are self-reported outcome measures used to assess patient's balance confidence and physical function, respectively. However, it is not known which outcome measure best reflects the ability to maintain balance in patients with THA and TKA. In validating outcome measures, they are usually compared with the gold standard which are typically the laboratory-based measures.

Force plate systems are laboratory-based systems that have made it possible to assess patients' balance quantitatively. Force plate systems record vertical forces acting on a platform to determine parameters for balance using center of pressure (CoP). ${ }^{3}$ Many force plate systems are available and commonly use three or four strain gauges. ${ }^{11}$ Researchers have used different balance assessment protocols to assess balance on force plate systems including quiet standing, ${ }^{12}$ perturbed standing, ${ }^{12}$ balance during functional tasks, ${ }^{13}$ and balance during eyes-open and eyes-closed conditions. ${ }^{14}$ However, the force plate systems are very expensive, time-consuming, not readily accessible, and impractical to use in assessing patients' balance on a day-to-day basis in clinical settings. Clinical outcome measures of balance, on the other hand, are cost-effective, easy to administer and score, readily available, and take relatively less time to administer and score. However, whether the clinical outcome measures provide comparable information as the force plate measure, in patients with THA and TKA, remains to be seen.
Therefore, the aim of the current study was to determine the relationship between selected clinically based outcome measures and laboratory-based force platform measure in patients following THA and TKA.

\section{Methods \\ Study design}

Participants in this study were a sample of convenience of consecutive patients referred to a large outpatient home physical therapy provider following either THA or TKA. Patients' charts were reviewed to assess their eligibility for the study. Patients who met the eligibility criteria were advised of the study. Eligible participants were visited by their physical therapist at their home on the first or second day after discharge from the hospital, which was about 7-10 days following their THA or TKA. Participants were assessed by their physical therapist for their eligibility and to collect relevant medical information. After the first visit, participants were visited once or twice a week until 5 weeks to impart postsurgical physical therapy and to monitor their progress. Following completion of the 5-week home physical therapy program, participants were advised to come to the biomechanics laboratory at the School of Physical Therapy.

When participants arrived at the biomechanics laboratory, they were given considerable time to get comfortable with the surroundings in the laboratory, following which they were advised about the procedure of the study. Subsequently, participants were assessed on four clinical outcome measures. First, participants were assessed on two selfreported outcome measures (the WOMAC and the $\mathrm{ABC}$ ), and then they were assessed on two clinician-administered outcome measures (the BBS and the TUG) in no particular order of administration. After assessments on these outcome measures, participants were then assessed on a laboratorybased outcome measure, the force plate. All participants were assessed on the clinical outcome measures as well as the laboratory-based outcome measure within a week (3-6 days) following the 5-week home physical therapy program. Ethics approval for the study was received from the University Research Ethics Board for Health Sciences Research Involving Human Subjects, University of Western Ontario, and all participants provided written consent prior to participation. One of the participants provided a written informed consent to have his picture taken on the laboratory-based outcome measure and the image published in the current study. This study is registered in ClinicalTrials.gov Protocol Registration System (ClinicalTrials.gov ID: NCT01555307; Protocol ID: R-06-461). 


\section{Participants and therapist}

All participants had primary unilateral THA or TKA as a result of hip or knee joint OA and had been advised by their surgeon to weight-bear as tolerated following their surgery. Patients with revision THA or TKA, or those who had been advised by their surgeon to remain non-weight-bearing or partial weight-bearing on either the operated or nonoperated leg, were excluded. Patients with neurological conditions, such as hemiplegia, peripheral neuropathy, Parkinson's disease, multiple sclerosis, and spinal cord compression, that might affect balance, and those who were considered unable to communicate or follow instructions were also excluded from the study.

Two physical therapists, who had 3-4 years of clinical experience as a physical therapist and who routinely provided physical therapy to patients who underwent THA and TKA, participated in the study. Assessments on the outcome measures were completed only by the primary investigator, who was one of the physical therapists.

\section{Clinical outcome measures}

\section{Berg Balance Scale}

The original 14-item BBS is a clinician-administered outcome measure that was originally designed to assess balance in the elderly and has been demonstrated to be valid, reliable, and responsive. ${ }^{7}$ It includes 14 simple balance tasks, which are scored on a five-point ordinal scale $(0,1,2,3$, and 4$)$, with a maximum total score of 56 . Higher scores on the BBS indicate better balance. All 14 performance tasks on the BBS focus on balance, and it has been previously used to assess balance in patients following hip fracture ${ }^{15}$ and knee OA. ${ }^{16}$

\section{The Timed Up and Go test}

The TUG is also administered by clinicians and is a test of functional mobility completed using a single chair and a $3 \mathrm{~m}$ walkway. It combines standing up from the chair, walking, turning around, and sitting down, and the performance is timed. The faster the time, the better is the subject's physical function. ${ }^{8}$ The test is simple, quick, requires no special equipment, and has been reported to be reliable, valid, and responsive for assessing functional mobility in an elderly population. ${ }^{17,18}$

\section{The Activities-specific Balance Confidence Scale}

The ABC is a self-reported outcome measure that was developed by Powell and Myers, ${ }^{9}$ and was demonstrated to be valid, reliable, and responsive to assess the level of patient's balance confidence in performing specific activities. The ABC has 16 items and is scored on a $0-100 \%$ response scale. An average of scores for all items is calculated as the patient's total $\mathrm{ABC}$ score. ${ }^{9}$ The $\mathrm{ABC}$ is simple and easy to understand, and takes 5-10 minutes to complete and score. The ABC has been used to assess balance confidence in patients with stroke, ${ }^{19}$ and to assess fall-related self-efficacy in elderly patients after hip fracture. ${ }^{20}$

\section{Western Ontario McMaster Universities Osteoarthritis Index}

The WOMAC is also a self-reported, disease-specific questionnaire used to assess patients having OA of the hip and/ or knee, and has been demonstrated to be valid, reliable, and responsive. ${ }^{10}$ It consists of 24 questions distributed among three subscales: 1) a pain subscale with five questions, 2) a stiffness subscale with two questions, and 3) a physical function subscale with 17 questions. The WOMAC allows patients to make their responses using five-point scales $(0,1$, 2, 3, and 4) with the total possible score being 96. Higher scores on the WOMAC indicate greater pain and stiffness, and greater difficulty in performing selected functional activities. ${ }^{10}$ While the WOMAC requests information from patients about their physical function rather than balance per se, it is very commonly used to assess patients with THA or TKA, and is widely popular among clinicians and patients. ${ }^{21-24}$ Although typically reported as a total score, the strong relationship between the pain and functional subscales of the WOMAC reported by Stratford et $\mathrm{al}^{24}$ suggests the use of function subscale score of the WOMAC in place of the total score. Subsequently, the WOMAC-function subscale (WOMAC-function) scores were used in all analyses in this study.

\section{Laboratory outcome measure Force plate}

Balance was assessed using an Advanced Mechanical Technology, Inc. (AMTI, Newton, MA, USA) stationary $51 \mathrm{~cm}$ $\times 46 \mathrm{~cm}$ steel force plate with implanted strain gauges, an amplifier, and a computer with Biosoft software (AMTI) which computed the CoP excursion in millimeters while the subject stood on the force plate. As the subject sways while standing on the force plate, the CoP excursion has trajectory in all directions (anterior-posterior and medial-lateral) of the sway which can be observed on the monitor of the computer. The Biosoft software computes an ellipse which covers $95 \%$ of the data points of the $\mathrm{CoP}$ excursions in square centimeters. The smaller the area of the $95 \%$ ellipse for a subject, the better is his/her balance. 
Previous studies have utilized CoP amplitude, which is the total distance traveled in the medial-lateral and anteriorposterior directions to describe CoP patterns during balance assessments. ${ }^{25,26}$ For the purposes of the current study, the 95\% ellipse area of the $\mathrm{CoP}$ amplitude in the medial-lateral and anterior-posterior directions was used as the CoP measure, and was calculated in square centimeters using the Biosoft software. The rationale given by Thapa et a ${ }^{14}$ for using an area measure to assess balance is that it represents the portion of the base of support utilized during standing conditions. The $95 \%$ ellipse area covers $95 \%$ of the data points of the $\mathrm{CoP}$ amplitude in the medial-lateral and anterior-posterior direction, which according to Lee and Park ${ }^{27}$ provides good information about balance in the anterior-posterior and medial-lateral directions, and is not biased by outliers. Swanenburg et $\mathrm{al}^{28}$ have reported a good reliability of $95 \%$ ellipse area of the CoP using AMTI force plate to assess balance on two-legged stance in elderly patients.

Standing balance was assessed on the force plate equipment placed in the same biomechanics laboratory. Participants were comfortably dressed in shirt and pants and were barefoot during the tests. Before the balance tests began, participants were comfortably seated on a chair placed behind the force plate while the testing procedure was demonstrated and reviewed. Participants were encouraged to ask any questions related to the procedure and report any fatigue they experience during the testing. The participants were provided with suitable rest periods if required.

A walker was positioned immediately in front of the force plate for safety purposes. The participants were instructed to use the arms of the chair to stand up and then to use the walker to achieve balance before each test (Figure 1). Once the participants obtained balance, they were asked to release the walker and stand in the test position for 10 seconds during the data capture interval. They were advised to use the walker in case they lost their balance. For safety reasons and to enhance the participant's confidence, another physical therapist was present by the participant's side to guard against any major loss of balance and potential fall. The 10-second data collection period began when the participants said "Now", indicating that they believed they had established balance.

The balance testing comprised three test conditions performed with the participant's eyes open. The three test conditions were 1) two-legged stance, 2) two-legged anterior lean stance, and 3) two-legged posterior lean stance. Testing started with the two-legged standing position with evenly distributed weight, followed by the anterior and posterior

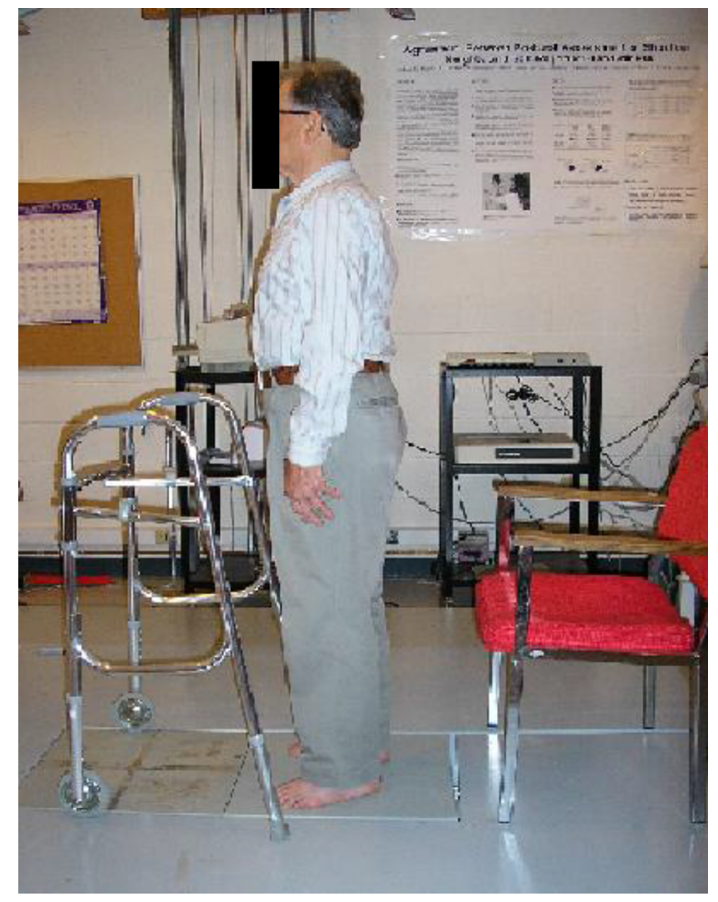

Figure I Participant in two-limb standing position on the force plate for balance testing.

lean standing positions. For the anterior and posterior lean stance, participants were instructed to lean anteriorly and posteriorly as far as they could comfortably and maintain the position. Two repetitions of each test were completed, with a 10 -second rest interval between repetitions during which the patient remained standing and used the walker for support, as needed. Demonstration of the balance tests on the force plate was provided to the participants by the primary investigator prior to balance testing. No trial training was given to the participants to avoid any learning effect.

\section{Statistical analysis}

Descriptive statistics were used to characterize participants with THA and TKA on the clinical measures and the force plate measure. Student's $t$-tests were used to compare participants who underwent THA with those who underwent TKA on the BBS, the TUG, the ABC, and the WOMAC-function clinical measures. Student's $t$-tests were also used to compare participants with THA to those with TKA on the $95 \%$ ellipse area of the force plate for the three test conditions (two-legged stance, anterior lean stance, and posterior lean stance). An average of scores of the two repetitions for each test condition was used as the final score of $95 \%$ ellipse area for that condition.

Pearson's correlation coefficients were used to compare scores on the BBS, the ABC, the TUG, and the WOMACfunction with those on the $95 \%$ ellipse area of the force plate 
for all the three test conditions. Also, Pearson's correlation coefficients were used to compare scores among the clinical measures. Correlations were also performed separately for participants with THA and those with TKA between the clinical measures and the $95 \%$ ellipse area of the force plate for the three test conditions.

Correlation coefficients were assessed using the criteria provided by Portney and Watkins. ${ }^{29}$ Correlation coefficients in the range of $0.00-0.25$ indicated little or no relationship, $0.25-0.50$ indicated a fair-to-moderate relationship, $0.50-0.75$ indicated a moderate-to-good relationship, and above 0.75 indicated a good-to-excellent relationship. All statistical tests were completed using STATISTICA, ${ }^{30}$ and the level of statistical significance was set at $p<0.05$.

\section{Sample size estimation}

Based on the hypothesized prediction of a significant and moderate correlation $(r \geq 0.40)$ between the BBS and the $95 \%$ ellipse area of the force plate, with a power of 0.80 at a significance level of 0.05 (two-tailed), 47 participants were required to participate in this study. ${ }^{31}$ Participants with THA and those with TKA were pooled together to achieve the required sample size for the study.

\section{Results}

A total of 63 participants participated in the study, of which 30 participants had THA and 33 participants had TKA. Thirty-five percent of the participants were males, and $65 \%$ females. Fifty-four participants completed the four clinical outcome measures (the BBS, the TUG, the ABC, and the WOMAC-function), and 50 participants completed the laboratory-based force plate outcome measure ( $95 \%$ ellipse area). Of the 54 participants who completed the clinical outcome measures, four patients were not tested on the force plate due to technical problems with the equipment. Demographic information of participants is presented in Table 1. No statistically significant differences were observed in age, height, and body mass between participants who had THA and those who had TKA $(p>0.05)$.
Table I Descriptive information of the participants

\begin{tabular}{lll}
\hline Surgery & THA & TKA \\
\hline Males $(\mathrm{n})$ & 12 & 10 \\
Females $(\mathrm{n})$ & 18 & 23 \\
Age $($ years $)$ & $68 \pm 8$ & $65 \pm 1 \mathrm{I}$ \\
Height $(\mathrm{cm})$ & $166 \pm 8$ & $166 \pm 1 \mathrm{I}$ \\
Mass $(\mathrm{kg})$ & $77 \pm 19$ & $86 \pm 20$ \\
Foot length $(\mathrm{cm})$ & $26 \pm 2$ & $26 \pm 2$ \\
Foot width $(\mathrm{cm})$ & $28 \pm 2$ & $29 \pm 1$ \\
\hline
\end{tabular}

Note: Values are mean \pm SD or $n$.

Abbreviations: THA, total hip arthroplasty; TKA, total knee arthroplasty; $\mathrm{SD}$, standard deviation.

Scores on clinical outcome measures are presented in Table 2 . No statistically significant differences were observed between participants who had THA and those who had TKA on the BBS, the TUG, the ABC, and the WOMAC-function $(p>0.05)$. Data on all clinical outcome measures were found to be normally distributed (Figure 2). Also, no statistically significant differences were observed between participants who had THA and those who had TKA on the $95 \%$ ellipse area for the three force plate test conditions - the two-legged stance, the anterior lean stance, and the posterior lean stance $(p>0.05$, Table 3$)$. Data on the force plate for all test conditions were found to be normally distributed (Figure 3).

Moderate correlations were observed between the BBS and the $95 \%$ ellipse area for all the three force plate test conditions ( $r=0.46-0.51$, Table 4$)$. The scatter plot in Figure 4 shows that the participants who scored near maximum on the BBS had the $95 \%$ ellipse area of the force plate varied within a considerably small part of the total range observed in the study sample. Fair correlations were observed between the TUG and the $95 \%$ ellipse area $(r=0.31-0.37)$, again for all the three force plate test conditions. However, low correlations were observed for the $\mathrm{ABC}$ and the WOMAC-function with the $95 \%$ ellipse area of the force plate $(r=0.11-0.25)$ on the three test conditions. Correlations performed separately for participants with THA and those with TKA also demonstrated a similar pattern of relationships between the clinical measures and the $95 \%$ ellipse area of the force plate as that observed for the pooled total participants.

Table 2 Means and SDs for the scores on clinical outcome measures

\begin{tabular}{lllll}
\hline Clinical measure & Total $(\mathbf{n}=\mathbf{5 4})$ & THA $(\mathbf{n}=\mathbf{2 6})$ & TKA $(\mathbf{n}=\mathbf{2 8})$ & $\mathbf{p}$ value \\
\hline BBS & $50.48 \pm 6.30$ & $50.48 \pm 5.37$ & $50.48 \pm 7.22$ & 1.00 \\
TUG & $13.92 \pm 4.98$ & $13.85 \pm 5.14$ & $14.00 \pm 4.90$ & 0.91 \\
ABC & $71.53 \pm 22.65$ & $75.62 \pm 18.94$ & $67.28 \pm 25.64$ & 0.18 \\
WOMAC-function & $14.83 \pm 11.26$ & $12.77 \pm 8.81$ & $16.88 \pm 13.12$ & 0.18 \\
\hline
\end{tabular}

Note: Values are mean \pm SD or $n$.

Abbreviations: SD, standard deviation; THA, total hip arthroplasty; TKA, total knee arthroplasty; BBS, Berg Balance Scale; TUG, Timed UP and Go test; ABC, Activitiesspecific Balance Confidence Scale; WOMAC-function, Western Ontario McMaster Universities Osteoarthritis Index-function subscale. 

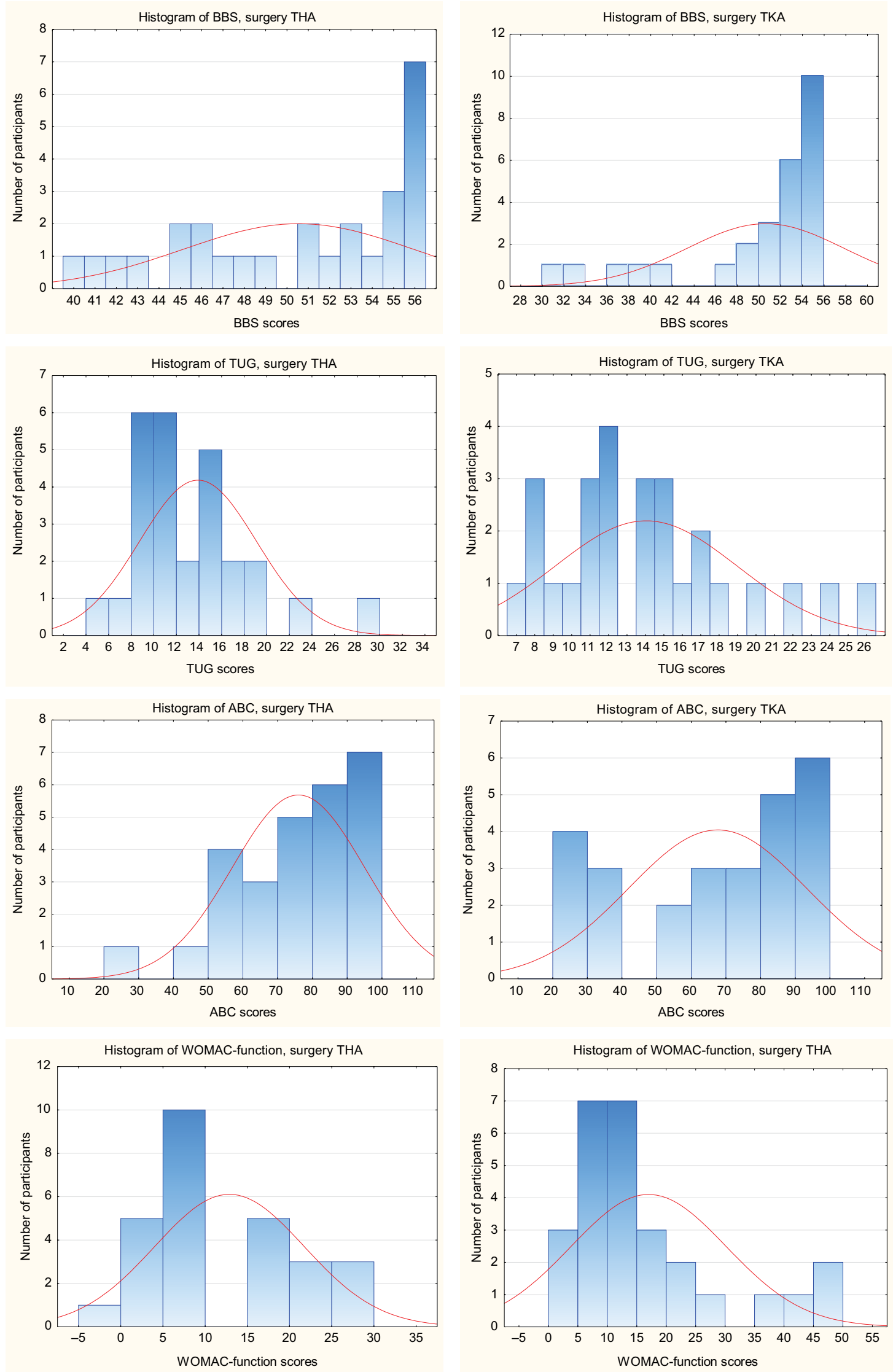

Figure 2 Histogram of scores on the clinical outcome measures, Berg Balance Scale (BBS), Timed Up and Go test (TUG), Activities-specific Balance Confidence Scale (ABC), and Western Ontario and McMaster Universities Osteoarthritis Index function subscale (WOMAC-function) by the type of surgery, total hip arthroplasty (THA), and total knee arthroplasty (TKA). 
Table 3 Values for $95 \%$ ellipse area force plate for the three test conditions

\begin{tabular}{lllll}
\hline Force plate test conditions & Total $(\mathbf{n}=\mathbf{5 0})$ & THA $(\mathbf{n}=\mathbf{2 4})$ & TKA $(\mathbf{n}=\mathbf{2 6})$ & $\mathbf{p}$-value \\
\hline Two-legged stance & $1.76 \pm 2.68$ & $1.60 \pm 1.65$ & $1.93 \pm 3.44$ & 0.66 \\
Anterior lean stance & $2.80 \pm 3.03$ & $3.07 \pm 2.63$ & $2.53 \pm 3.43$ & 0.53 \\
Posterior lean stance & $3.56 \pm 3.94$ & $3.86 \pm 3.44$ & $3.25 \pm 4.44$ & 0.58 \\
\hline
\end{tabular}

Note: Values are mean $\pm S D$, or $n$.

Abbreviations: SD, standard deviation; THA, total hip arthroplasty; TKA, total knee arthroplasty.
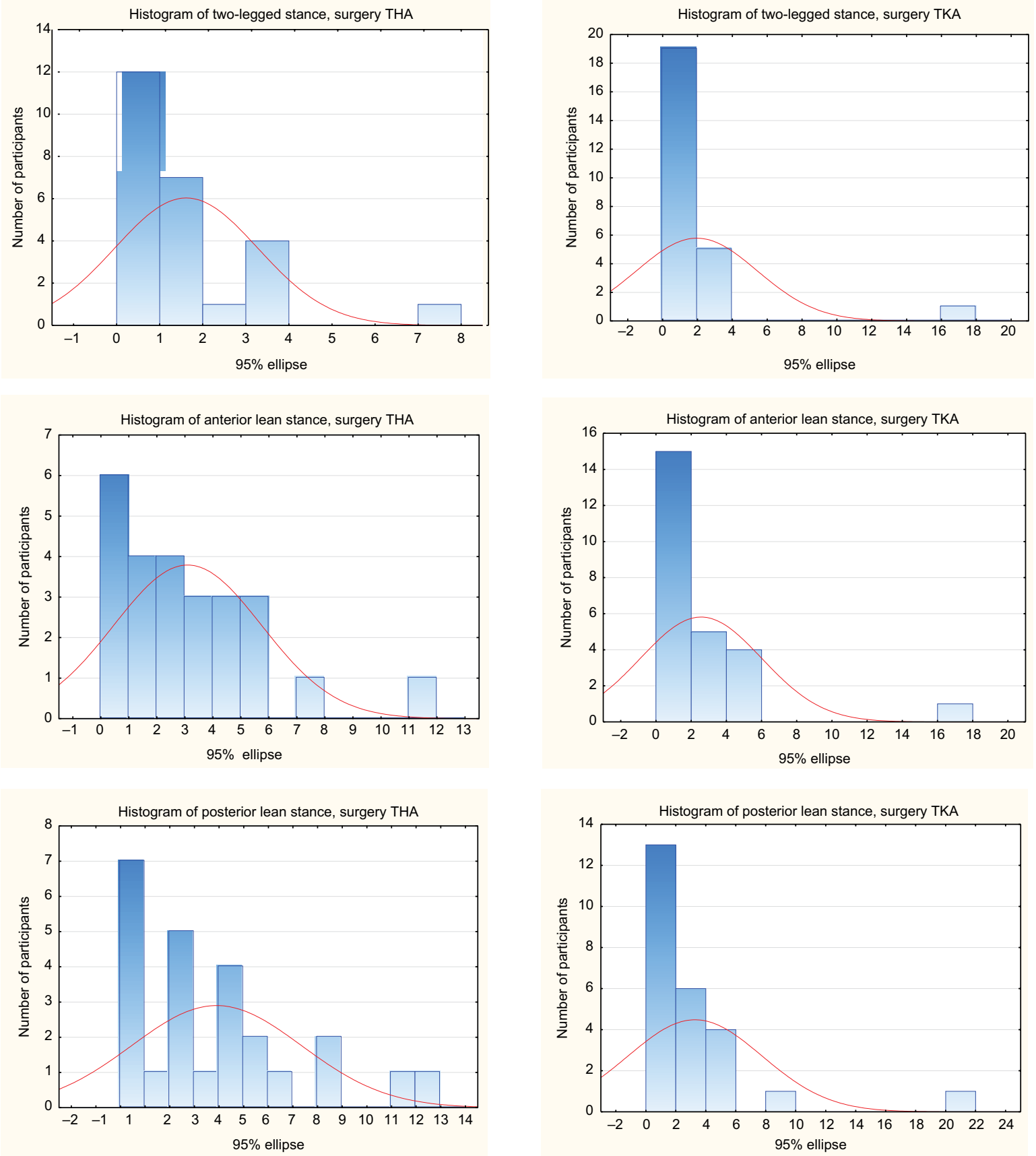

Figure 3 Histogram of scores on the force plate for the different test conditions by the type of surgery, total hip arthroplasty (THA) and total knee arthroplasty (TKA). 
Table 4 Correlations between the clinical outcome measures and the $95 \%$ ellipse area of the force plate for the three test conditions $(p<0.05)$

\begin{tabular}{lllll}
\hline Force plate test & \multicolumn{4}{c}{ Clinical measures $(\mathbf{n}=\mathbf{5 0})$} \\
\cline { 2 - 5 } conditions & BBS & TUG & ABC & WOMAC-function \\
\hline Two-legged stance & $-0.5 \mathrm{I}$ & 0.35 & -0.25 & 0.25 \\
Anterior lean stance & -0.46 & 0.31 & -0.16 & 0.11 \\
Posterior lean stance & -0.46 & 0.37 & -0.24 & 0.24
\end{tabular}

Abbreviations: BBS, Berg Balance Scale; TUG, Timed Up and Go test; $A B C$, Activities-specific Balance Confidence Scale; WOMAC-function, Western Ontario McMaster Universities Osteoarthritis Index-function subscale.

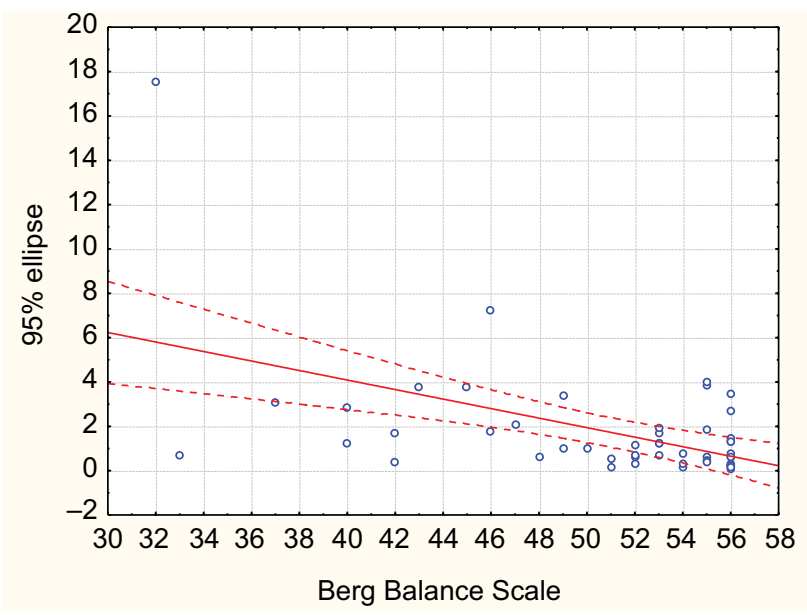

Figure 4 Scores on the Berg Balance Scale plotted against 95\% ellipse area of the force plate. Regression line shown as illustration $(r=-0.5 \mathrm{I})$.

Table 5 Correlations among the four clinical measures $(p<0.05)$

\begin{tabular}{lllll}
\hline Clinical measure & BBS & TUG & ABC & WOMAC-function \\
\hline BBS & 1.00 & -0.75 & 0.60 & -0.68 \\
TUG & & 1.00 & -0.73 & 0.66 \\
ABC & & & 1.00 & -0.70 \\
WOMAC-function & & & & 1.00
\end{tabular}

Abbreviations: BBS, Berg Balance Scale; TUG, Timed UP and Go test; ABC, Activities-specific Balance Confidence Scale; WOMAC-function, Western Ontario McMaster Universities Osteoarthritis Index-function subscale.

Correlations among the four clinical measures are presented in Table 5. The BBS and the TUG demonstrated a good relationship ( $r=-0.75)$. While the ABC demonstrated a good relationship with the TUG $(r=-0.73)$, the relationship with the BBS was moderate $(r=0.60)$. The WOMAC-function demonstrated only moderate relationships with the BBS, the TUG, and the ABC $(r=0.66-0.70)$.

\section{Discussion}

The current study explored relationships of the clinical outcome measures the BBS, the TUG, the ABC, and the WOMAC-function with the laboratory-based force plate measure (95\% ellipse area). The authors used concurrent validity, a measure of criterion-related validity, by comparing the clinical measures of balance with a force plate measure that has been established as standard for assessment of balance. ${ }^{29}$ Moderate correlations of the BBS with the $95 \%$ ellipse area of the force plate suggest that the BBS provides information about the ability to maintain balance and supports its validity in assessing balance in patients with THA and TKA. The BBS was the only measure among the four outcome measures in the current study that demonstrated the strongest relationship with the $95 \%$ ellipse area of the force plate.

The BBS has been previously compared with other different force plate measures of balance but not the $95 \%$ ellipse area, and in different sample populations but not in patients with THA and TKA.

Frykberg et al ${ }^{32}$ reported correlations similar to that observed in the current study between the BBS subgroup score of items that involved maintaining a position and the force plate measure in patients who had stroke $(r=-0.50$, $p<0.05$ ), and they used the mean velocity of CoP displacement as a force plate measure. Although the mean velocity of CoP displacement has been identified as a consistent and stable force plate measure, ${ }^{12,33}$ Frykberg et al ${ }^{32}$ reported a disproportionably low mean velocity of $\mathrm{CoP}$ displacement in participants even with considerably good balance with a maximum score on the BBS. However, in the current study, as illustrated in Figure 4, the 95\% ellipse area of the force plate could be quite small in participants with considerable balance ability as demonstrated by their maximum scores on the BBS.

Sabchuk et a $l^{34}$ have also reported fair-to-moderate correlations between the BBS and the force plate measure in young and elderly healthy individuals $(r=-0.37$ to $-0.51, p<0.05$ ) using CoP displacement as a force plate measure, and under different test conditions such as eyes-open and eyes-closed conditions. Berg et al ${ }^{12}$ reported fair correlations between the BBS and the force plate measure during platform-induced sway $(r=-0.38, p<0.05)$, and moderate correlations when the platform was stationary $(r=-0.55, p<0.05)$ using CoP amplitude and speed as force plate measure, but the participants in the study were healthy elderly individuals. Based on the results of the current study, the authors support the use of the BBS by clinicians for assessment of balance in patients with THA and TKA.

The TUG demonstrated fair correlations with the $95 \%$ ellipse area of the force plate. Previous research by Sabchuk et $\mathrm{al}^{34}$ that compared the TUG with $\mathrm{CoP}$ displacement measure 
of force plate also reported fair-to-moderate correlations $(r=0.32-0.47, p<0.05)$ in young and elderly volunteers. While the TUG demonstrated good correlations with the BBS in the current study, Sabchuk et $\mathrm{al}^{34}$ reported moderate correlations $(r=-0.57, r<0.05)$ between the TUG and the BBS. All participants in the current study were elderly, while one half of the participants in the study by Sabchuk et $\mathrm{al}^{34}$ were young and the other half elderly. Consistent with the results of the current study, Hatch et $\mathrm{al}^{35}$ reported good correlations between the TUG and the BBS ( $r=0.81$, $p<0.01)$ in community-dwelling elderly individuals. The results of the current study suggest that if the BBS is not available, the TUG may also be used to assess balance in patients with THA and TKA and can provide comparable information as that of the force plate measure and the BBS.

The ABC demonstrated low correlations with the $95 \%$ ellipse area of the force plate in the current study and moderate correlations with the BBS. Previous work by Lajoie et $\mathrm{al}^{36}$ has also reported low correlations $(r=-0.17, p<0.05)$ for the anteroposterior sway of the force plate measure and moderate correlations $(r=-0.37, p<0.05)$ for the lateral sway of the force plate measure with the $\mathrm{ABC}$ in elderly participants. The same authors also reported moderate correlations between the ABC and the BBS $(r=0.67, p<0.05)^{36}$ similar to the results observed in the current study. On the other hand, the ABC demonstrated good correlations with the TUG in the current study. Hatch et al, ${ }^{35}$ however, reported moderate correlations between the ABC and the TUG $(r=0.69$, $p<0.05)$ and good correlations $(r=0.75, p<0.05)$ between the $\mathrm{ABC}$ and the BBS, but the participants in the study were healthy community-dwelling elderly individuals. The result of the current study suggests that the $\mathrm{ABC}$ may not provide accurate information on balance in patients with THA and TKA. The ABC is a self-reported measure that may provide information on patients' confidence about their balance but may not provide information about patients' balance per se.

The WOMAC-function correlated the least with the 95\% ellipse area among the four clinical measures used in the current study, and the correlations were low. In addition, the WOMAC-function correlated moderately with the BBS, the TUG, and the ABC. The WOMAC-function, like the ABC, is also a self-reported measure developed to assess pain, stiffness, and physical function in patients with THA and TKA, and not necessarily balance. Low correlations were expected between the WOMAC-function and the 95\% ellipse area of the force plate. The authors were trying to explore the ability of the WOMAC to provide any information on balance in addition to function as it is very widely used by clinicians to assess patients with THA and TKA. Although very popular among clinicians to assess patients with THA and TKA, the WOMAC has not been studied for its relationship with outcome measures of balance in the past. Results of the current study suggest that the WOMAC-function does not provide any information on balance in patients with THA and TKA, and does not support its use to assess balance in these patients.

In the current study, no differences were observed between patients with THA and those with TKA on all the outcome measures, and data on patients with THA and TKA were pooled together for the purpose of the study. Previous studies have also reported no differences in functional outcomes between patients who had undergone THA and those who had undergone TKA at 5-7 weeks ${ }^{37}$ and at 6 months ${ }^{38,39}$ postsurgery.

In conclusion, the current study provided information suggesting that the BBS is preferable for assessing balance in patients with THA and TKA over the TUG and the ABC. The $\mathrm{ABC}$ and the WOMAC-function may not provide any information on the balance ability of these patients and may not be used to assess balance. Moderate relationship was observed between the BBS and the 95\% ellipse area of the force plate. Fair relationship was observed between the TUG and the $95 \%$ ellipse area, and good relationship between the TUG and the BBS. The relationship of the $\mathrm{ABC}$ and the WOMAC-function with the $95 \%$ ellipse area of the force plate was weak.

This study presented some limitations. Participants in the study were a sample of convenience referred to homebased community physical therapy within a limited geographical area. Although participants were provided with adequate rest periods, influence of participants' fatigue on their performance on outcome measures, especially during the later stages of the study procedure, cannot be ruled out. The clinical and force plate measures were administered to patients simultaneously one after the other, and how much learning effect occurred in participants as the assessment progressed is unclear.

\section{Acknowledgments}

The authors would like to thank all the participants who took part in this study.

\section{Disclosure}

The authors report no conflicts of interest in this work. 


\section{References}

1. Brouwer B, Musselman K, Culham E. Physical function and health status among seniors with and without a fear of falling. Gerontology. 2004;50(3):135-141.

2. Cho BL, Scarpace D, Alexander NB. Tests of stepping as indicators of mobility, balance, and fall risk in balance-impaired older adults. $\mathrm{J} \mathrm{Am}$ Geriatr Soc. 2004;52(7):1168-1173.

3. Riemann BL, Guskiewicz KM, Sheilds EW. Relationship between clinical and forceplate measures of postural stability. J Sport Rehabil. 1999;8(2):71-82.

4. Fridén T, Zätterström R, Lindstrand A, Moritz U. A stabilometric technique for evaluation of lower limb instabilities. Am J Sports Med. 1989;17(1):118-122.

5. Wegener L, Kisner C, Nichols D. Static and dynamic balance responses in persons with bilateral knee osteoarthritis. J Orthop Sports Phys Ther. 1997;25(1):13-18.

6. Mitchell SY,McCaskieAW, Francis RM, Peaston RT, BirrellFN, LingardEA. P69 falls in patients with hip and knee osteoarthritis: the impact of joint replacement surgery. J Bone Joint Surg (Br). 2008;90 (Suppl B):385.

7. Berg K, Wood-Dauphine S, William JI, Gayton D. Measuring balance in the elderly: preliminary development of an instrument. Physiother Can. 1989;41(6):304-311.

8. Podsiadlo D, Richardson S. The timed "Up \& Go": a test of basic functional mobility for frail elderly persons. J Am Geriatr Soc. 1991;39(2):142-148.

9. Powell LE, Myers AM. The Activities-specific Balance Confidence (ABC) Scale. J Gerontol A Biol Sci Med Sci. 1995;50A(1):M28-M34.

10. Bellamy N, Buchanan WW, Goldsmith H, Campbell J, Stitt L. Validation study of WOMAC: a health status instrument for measuring clinically-important patient-relevant outcomes following total hip or knee arthroplasty in osteoarthritis. J Orthop Rheumat. 1988;1:95-108.

11. Bauer C, Gröger I, Rupprecht R, Gassmann KG. Intrasession reliability of force platform parameters in community-dwelling older adults. Arch Phys Med Rehabil. 2008;89(10):1977-1982.

12. Berg KO, Maki BE, Williams JI, Holliday PJ, Wood-Dauphinee SL. Clinical and laboratory measures of postural balance in an elderly population. Arch Phys Med Rehabil. 1992;73(11):1073-1080.

13. Karst GM, Venema DM, Roehrs TG, Tyler AE. Center of pressure measures during standing tasks in minimally impaired persons with multiple sclerosis. J Neural Phys Ther. 2005;29(4):170-180.

14. Thapa PB, Gideon P, Brockman KG, Fought RL, Ray WA. Clinical and biomechanical measures of balance as fall predictors in ambulatory nursing home residents. J Gerontol A Biol Sci Med Sci. 1996;51(5):M239-M246.

15. Kulmala J, Sihvonen S, Kallinen M, Alen M, Kiviranta I, Sipilä S. Balance confidence and functional balance in relation to falls in older persons with hip fracture history. J Geriatr Phys Ther. 2007;30(3):114-120.

16. Sun SF, Hsu CW, Hwang CW, et al. Hyaluronate improves pain, physical function and balance in the geriatric osteoarthritic knee: a 6-month followup study using clinical tests. Osteoarthritis Cartilage. 2006;14(7):696-701.

17. Berger L, Klein C, Commandeur M. Evaluation of the immediate and midterm effects of mobilization in hot spa water on static and dynamic balance in elderly subjects. Ann Readapt Med Phys. 2008;51(2):84-95.

18. Bouillon LE, Sklenka DK, Driver AC. Comparison of training between 2 cycle ergometers on dynamic balance for middle-aged women. J Sport Rehabil. 2009;18(2):316-326.

19. Botner EM, Miller WC, Eng JJ. Measurement properties of the Activities-specific Balance Confidence Scale among individuals with stroke. Disabil Rehabil. 2005;27(4):156-163.
20. Petrella RJ, Payne M, Myers A, Overend T, Chesworth B. Physical function and fear of falling after hip fracture rehabilitation in the elderly. Am J Phys Med Rehabil. 2000;79(2):154-160.

21. Moffet H, Collet JP, Shapiro SH, Paradis G, Marquis F, Roy L. Effectiveness of intensive rehabilitation on functional ability and quality of life after first total knee arthroplasty: a single-blind randomized controlled trial. Arch Phys Med Rehabil. 2004;85(4):546-556.

22. Kramer JF, Speechley M, Bourne R, Rorabeck C, Vaz M. Comparison of clinic and home-based rehabilitation programs after total knee arthroplasty. Clin Orthop Relat Res. 2003;(410):225-234.

23. Sashika H, Matsuba Y, Watanabe Y. Home program of physical therapy: effect on disabilities of patients with total hip arthroplasty. Arch Phys Med Rehabil. 1996;77(3):273-277.

24. Stratford PW, Dogra M, Woodhouse L, Kennedy DM, Spadoni GF. Validating self-report measures of pain and function in patients undergoing hip and knee arthroplasty. Physiother Can. 2009;61(4):189-194.

25. Giemza C, Ostrowska B, Matczak-Giemza M. The effect of physiotherapy training programme on postural stability in men with hip osteoarthritis. Aging Male. 2007;10(2):67-70.

26. Arokoski JP, Leinonen V, Arokoski MH, Aalto H, Valtonen H. Postural control in male patients with hip osteoarthritis. Gait Posture. 2006;23(1):45-50.

27. Lee G, Park AE. Development of a more robust tool for postural stability analysis of laparoscopic surgeons. Surg Endosc. 2008;22(4): 1087-1092.

28. Swanenburg J, de Bruin ED, Favero K, Uebelhart D, Mulder T. The reliability of postural balance measures in single and dual tasking in elderly fallers and non-fallers. BMC Musculoskelet Disord. 2008; 9:162.

29. Portney LG, Watkins MP. Foundations of Clinical Research. 2nd ed. Upper Saddle River, NJ: Prentice-Hall Inc; 2000.

30. StatSoft Inc. STATISTICA 7.0. Tulsa, OK; StatSoft Inc.: 2000.

31. Hulley SB, Cummings SR, Browner WS, Grady D, Hearst N, Newman TB. Designing Clinical Research. 2nd ed. Philadelphia, PA: Lippincott Williams \& Wilkins; 2001.

32. Frykberg GE, Lindmark B, Lanshammar H, Borg J. Correlation between clinical assessment and force plate measurement of postural control after stroke. J Rehabil Med. 2007;39(6):448-453.

33. Stevenson TJ, Garland SJ. Standing balance during internally produced perturbations in subjects with hemiplegia: validation of the balance scale. Arch Phys Med Rehabil. 1996;77(7):656-662.

34. Sabchuk RAC, Bento PCB, Rodacki ALF. Comparison between field balance tests and force platform. Rev Bras Med Esporte. 2012;18(6):404 408.

35. Hatch J, Gill-Body KM, Portney LG. Determinants of balance confidence in community-dwelling elderly people. Phys Ther. 2003;83(12): 1072-1079

36. Lajoie Y, Girard A, Guay M. Comparison of the reaction time, the Berg Scale and the ABC in non-fallers and fallers. Arch Gerontol Geriatr. 2002;35(3):215-225.

37. Jogi P, Overend TJ, Spaulding SJ, Zecevic A, Kramer JF. Effectiveness of balance exercises in the acute post-operative phase following total hip and knee arthroplasty: a randomized clinical trial. SAGE Open Med. 2015;3:2050312115570769.

38. Jones CA, Voaklander DC, Johnston DW, Suarez-Almazon ME. The effect of age on pain, function and quality of life after total hip and knee arthroplasty. Arch Intern Med. 2001;161(3):454-460.

39. Mahomed NN, Liang MH, Cook EF, et al. The importance of patient expectations in predicting functional outcomes after total joint arthroplasty. J Rheumatol. 2002;29(6):1273-1279. 
Orthopedic Research and Reviews is an international, peer-reviewed, open access journal that focusing on the patho-physiology of the musculoskeletal system, trauma, surgery and other corrective interventions to restore mobility and function. Advances in new technologies, materials, techniques and pharmacological agents are particularly welcome. The manuscript management system is completely online and includes a very quick and fair peer-review system, which is all easy to use. Visit http://www.dovepress.com/testimonials.php to read real quotes from published authors.

Submit your manuscript here: https://www.dovepress.com/orthopedic-research-and-reviews-journal 